\section{Biodiversity data are out of local taxonomists' reach}

SIR - Exchange of information about biodiversity is mandated by the legally binding international Convention on Biological Diversity, as are monitoring and benefit sharing. Yet researchers in the developing world, where most of the biodiversity is found, are unable to access much of this information. This impedes the monitoring of biodiversity: monitoring depends on the proper identification of species, and this is hindered by a lack of both specialists and access to relevant taxonomic information.

The number of online publications with taxonomic content is increasing, and online tools are becoming available to mash up taxonomic with other information, for example at ispecies.org (see "Mashups mix data into global service" Nature 439, 6-7; 2006). But copyright and high costs put this information beyond the reach of many in the developing world - which is home to more than $95 \%$ of species whose descriptions have been published. More than half the 1,600 descriptions of new ant species published in the past ten years are copyrighted, for example, but none are in journals published in the developing world (see antbase.org).

This seems little better than biopiracy: taking biodiversity material from the developing world for profit, without sharing benefit or providing the people who live there with access to this crucial information.

A simple solution would be to treat species descriptions as we do gene sequences, and have them openly accessible. Open-access descriptions of new species could then be a mandatory factor in making them valid under the various codes of biological nomenclature. A recent Commentary by Andrew Polaszek and colleagues ("A universal register for animal names" Nature 437,$477 ; 2005$ ) describes how the International Commission on Zoological Nomenclature proposes to facilitate this process for animal descriptions, through a register called ZooBank. However, present copyright laws prevent the mandatory inclusion of what would be an immensely useful piece of information, the actual description of the species.

Donat Agosti

American Museum of Natural Histary, New York, New York 10024-5192, USA

\section{No clear evidence to disprove optics thesis}

SIR — David G. Stork, in his review of the book Optics, Instruments and Painting 1420-1720: Reflections on the Hockney-Falco Thesis ("Tracing the history of art" Nature
$438,916-917 ; 2005)$ reports the claim that appropriate concave mirrors to project optical images onto a canvas for tracing could not have existed in the fifteenth century. He concludes that the book may "close the door on the Hockney-Falco tracing thesis".

But our thesis is about the use of optics, not necessarily concave mirrors (although there is strong circumstantial evidence for mirrors). David Hockney and I have explicitly written during the past five years that none of the optical evidence we have found allows us to distinguish between the use of refractive versus reflective optics; either or both types are possibilities.

The spectacles and the magnifying glass in Tommaso da Modena's 1351 frescos would each have been able to project appropriate images, as would the spectacles in van Eyck's van der Paele altarpiece of about 1435. Charles M. Falco

College of Optical Sciences, G-SBuilding 77, University of Arizona, Tucson,

Arizona 85721-0077, USA

\section{Branding can be justified in vital conservation research}

SIR — Your News Story "Animal-rights group sues over 'disturbing' work on sea lions ${ }^{\text {" }}$ (Nature 436, 315; 2005) shows how wildlife researchers face escalating criticism over their use of invasive procedures to collect ecological data for population management. Although we appreciate and agree that all due consideration should be paid to the welfare of study animals, we argue that invasive research techniques are sometimes inevitable and integral parts of animal research in the global pursuit of biodiversity conservation.

The branding of seals is one technique criticized for being an invasive conservation tool. The technique has, however, provided important insights on juvenile survival that help to explain the processes involved in recent population declines - and a longterm programme of branding southern elephant seals (Mirounga leonina) on Macquarie Island, Australia, has shown that it has no discernable long-term impact on survival or condition (C. R. McMahon et al. J. Wildl. Mgmt, in the press).

The issue of branding on Macquarie Island became so controversial that the programme was suspended indefinitely (J. A. JabourGreen and C. J. A. Bradshaw J. Nat. Conserv. $12,25-39 ; 2004)$. Similar intervention has occurred in conservation research on two endangered species: New Zealand sea lions (Phocarctos hookeri) in New Zealand and Steller sea lions (Eumetopias jubatus) in the United States. Such political interference on the basis of animal-welfare issues is difficult to justify, considering that, in the United States alone, some 36 million beef cattle are hot- or cryo-branded each year, according to the US Department of Agriculture.

Effective conservation of species is urgently needed in the current biodiversity crisis. To this end, we urge scientists and welfare lobbyists to combine their efforts and consider objectively the pertinent issues balancing the desperate need for sound biological information with animal welfare. C.R. McMahon*, C. J.A. Bradshawt, G.C. Hays* *Institute of Environmental Sustainability, School of Biological Sciences, University of Wales Swansea, Singleton Park, Swansea SA2 8PP, UK †School for Environmental Research, Institute of Advanced Studies, Charles Darwin University, Darwin, Northern Territory 0909, Australia

\section{Don't forget randomness is still just a hypothesis}

SIR - Anton Zeilinger's bold essay "The message of the quantum " Nature 438, 743; 2005) claims "the discovery that individual events are irreducibly random is probably one of the most significant findings of the twentieth century." But we should not forget that the claim of true randomness has not yet been backed by evidence. Neither Heisenberg's uncertainty principle nor Bell's inequality exclude the possibility, however small, that the Universe, including all observers inhabiting it, is in principle computable by a completely deterministic computer program, as first suggested by computer pioneer Konrad Zuse in 1967 (Elektron. Datenverarb. 8, 336-344; 1967).

The principle of Occam's razor, which is fundamental to theory-building, favours simple explanations (describable by few bits of information) over complex ones. But if the Universe's history really included many truly random events, an enormous amount of information would be necessary to describe all the random observations inexplicable by the known, simple, elegant, compactly describable laws of physics.

A few previous attempts at discovering a pseudo-random generator behind seemingly random physical events have failed (see T. Erber and S. Putterman Nature 318, $41-43 ; 1985)$. But as long as the randomness hypothesis has not been verified, physicists should keep trying to falsify it and search not only for statistical laws but also for deterministic rules explaining any type of hitherto unexplained apparent randomness. Juergen Schmidhuber IDSIA, Galleria 2, 6928 Manno-Lugano, Switzerland and

Robotics and Embedded Systems, Technische Universităt München, Boltzmannstraße 3 , 85748 Garching bei München, Germany

Contributions to Correspondence may be submitted to corres@nature.com. 